\title{
Low Temperature Growth of Single-Walled Carbon
}

\section{Nanotubes by Plasma Enhanced Chemical Vapor}

\section{Deposition}

Eun Ju Bae , Yo-Sep Min , Donghun Kang, Ju-Hye Ko , and Wanjun Park*

Materials \& Devices Research Center, Samsung Advanced Institute of Technology, Yong-In, Kyeongki-

$$
\text { Do, 449-712, Korea }
$$

E-mail: wanjun@samsung.com

\section{SUPPORTING INFORMATION}

A schematic diagram of remote-PECVD system (Fig. S1) and a low magnification TEM image (Fig. S2) of the grown nanotubes are available. 


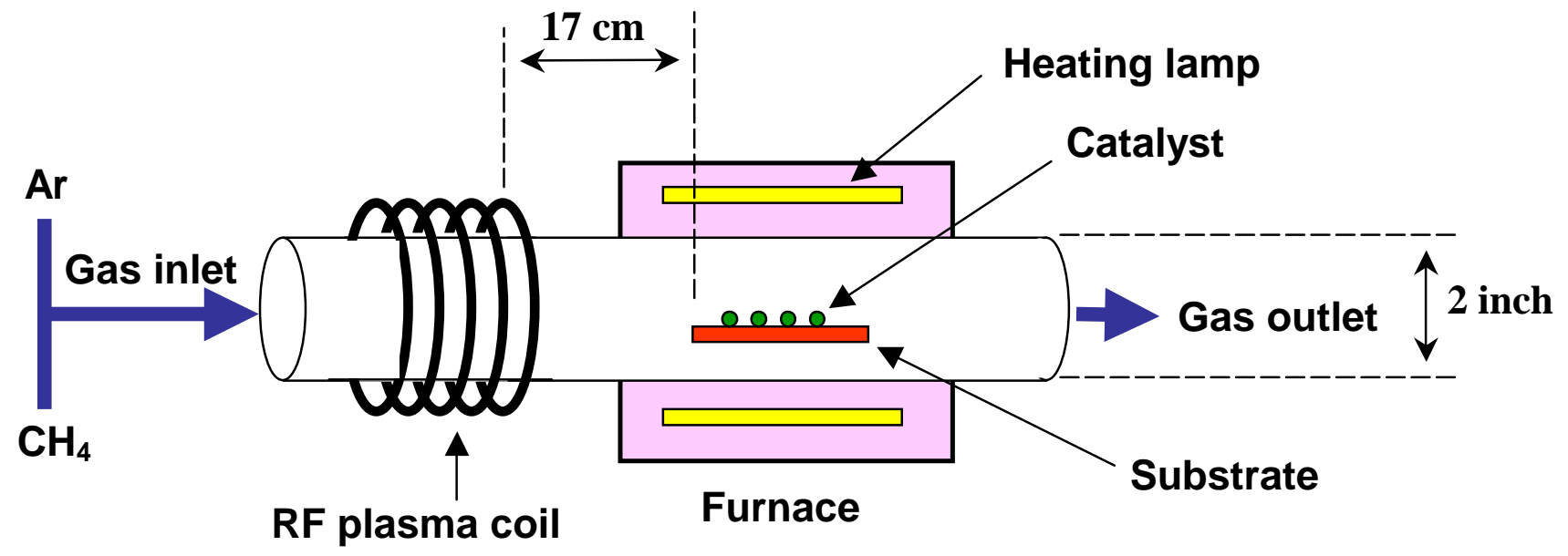

Figure S1. A schematic diagram of the remote PECVD system used in this work. 


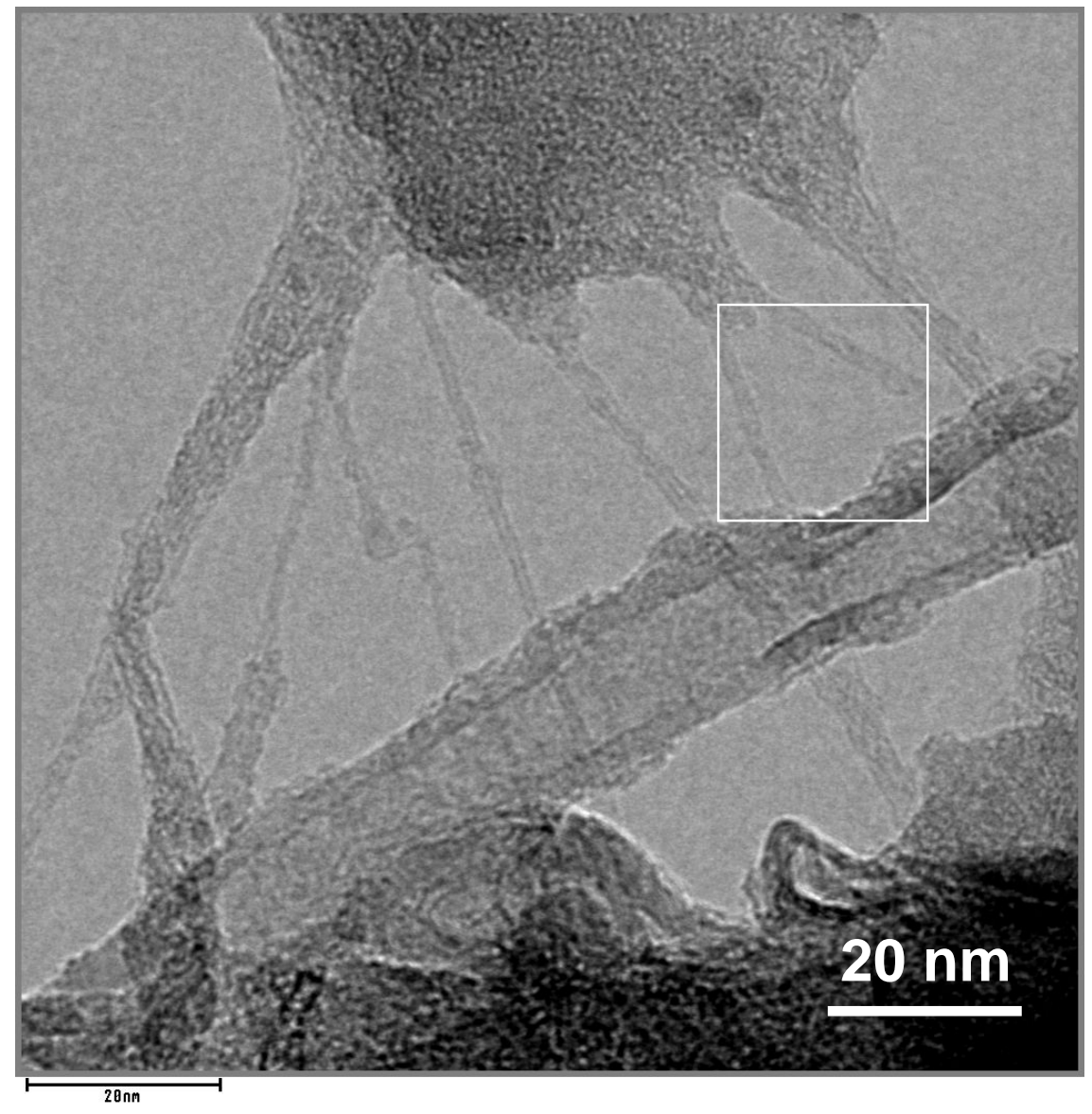

Figure S2. Low magnification TEM image of the grown nanotubes. Figure $8 \mathrm{~d}$ is the high resolution TEM image of the area designated with a box. 